\title{
Ab Initio Theory of Polar Semiconductor Surfaces. I. Methodology and the (22) Reconstructions of GaAs(111)
}

\section{Citation}

Kaxiras, Efthimios, Yaneer Bar-Yam, John D. Joannopoulos, and K.C. Pandey. 1987. Ab initio theory of polar semiconductor surfaces. I. Methodology and the (22) reconstructions of GaAs(111). Physical Review Series B 35, no. 18: 9625-9635.

\section{Published Version}

http://dx.doi.org/10.1103/PhysRevB.35.9625

\section{Permanent link}

http://nrs.harvard.edu/urn-3:HUL.InstRepos:10605457

\section{Terms of Use}

This article was downloaded from Harvard University's DASH repository, and is made available under the terms and conditions applicable to Other Posted Material, as set forth at http:// nrs.harvard.edu/urn-3:HUL.InstRepos:dash.current.terms-of-use\#LAA

\section{Share Your Story}

The Harvard community has made this article openly available.

Please share how this access benefits you. Submit a story.

\section{Accessibility}




\author{
E. Kaxiras, Y. Bar-Yam, and J. D. Joannopoulos \\ Department of Physics, Massachusetts Institute of Technology, Cambridge, Massachusetts 02139 \\ K. C. Pandey \\ IBM Thomas J. Watson Research Center, P.O. Box 218, Yorktown Heights, New York 10598
}

(Received 20 January 1987)

\begin{abstract}
A methodology is developed for the theoretical study of the polar surfaces of compound semiconductors. It is based on the calculation of the total energy in the context of density-functional theory in the pseudopotential approximation. The method is used to investigate the $(2 \times 2)$ reconstructions of GaAs(111). Emphasis is given to the relative chemical potential, which plays a crucial role in determining the lowest-energy geometry for surfaces with different stoichiometries. The totalenergy versus chemical-potential curves indicate that there are at least two stable reconstructions. We predict one to be an As-triangle geometry and the other the Ga vacancy.
\end{abstract}

\section{INTRODUCTION}

In the past several years, a great deal of attention has been devoted to polar surfaces of technologically important binary semiconductors, of which representative examples are the (111) (Ga-terminated) and (1 $\overline{1} \overline{1}$ ) (Asterminated) surfaces of GaAs. Such surfaces are not exposed by cleavage and must be grown by elaborate molecular-beam-epitaxy (MBE) techniques. ${ }^{1-3}$ Diffraction experiments, such as low-energy electron diffraction ${ }^{4}$ (LEED) and synchrotron $\mathrm{x}$-ray diffraction, ${ }^{5}$ have provided detailed studies of the surface reconstruction patterns. There is strong evidence from the experiments for the existence of nonstoichiometric surface reconstructions, ${ }^{4,5}$ as well as indications that the chemical potentials (determined by growth conditions $)^{1,6}$ play an important role. Several issues are still unresolved however. The rich variety of patterns observed, such as $(2 \times 2),(\sqrt{3} \times \sqrt{3)}$, $(3 \times 3)$, and $(\sqrt{19} \times \sqrt{19})$, correspond to various atomic arrangements about which very little is known. Even in the case of the widely accepted Ga-vacancy model ${ }^{4,7}$ for the $(111)(2 \times 2)$ surface, it is not well understood how this geometry can be stable, despite the fact that it is an Asrich reconstruction on the Ga-terminated surface; this seems to be contradicted by the exceedingly high (almost unity) sticking coefficient of Ga observed in growth experiments. ${ }^{1}$ A similar $(2 \times 2)$ pattern is observed on the $(\overline{1} \overline{1} \overline{1})$ surface, but there is no well-established model for this case, ${ }^{8}$ and no clear notion of what drives the complicated transition between this reconstruction and the Gastabilized $(\sqrt{19} \times \sqrt{19})$ reconstruction. ${ }^{1,3}$

The present work is aimed at resolving the above questions from a theoretical total-energy-minimization point of view, and exploring a variety of possible reconstructions to gain a better understanding of the complex surface phenomena observed.

The surface reconstructions of binary compound semiconductors present a challenging theoretical problem for two reasons, both associated with the existence of different kinds of atoms. The first reason is the polar character of the surfaces, which arises when only one of the two kinds of atoms is exposed at the surface plane; this polarity introduces complications to the conventional computational schemes such as slab calculations. The other theoretical difficulty comes from the possibility of nonstoichiometric surface reconstructions; this allows for a wide variety of atomic configurations and requires the knowledge of the chemical potentials of the two species of atoms with respect to the surface.

Both of these difficulties were overcome in the context of $a b$ initio pseudopotential calculations of the total energy. Previous theoretical work has been restricted to semiempirical tight-binding calculations by Nishida ${ }^{9}$ and by Chadi, ${ }^{7,8}$ which lack the self-consistency and high accuracy of the present approach. They also were of a restricted nature, since in one case (Nishida) only $(1 \times 1)$ surfaces were examined, and in the other (Chadi) the relative chemical potential was not taken into account. Here we examine the $(2 \times 2)$ reconstructions of the (111) and $(\overline{1} \overline{1} \overline{1})$ surfaces of GaAs, although higher-order reconstructions, e.g., $(3 \times 3)$, and $(\sqrt{19} \times \sqrt{19})$, are also observed experimentally. The search over many atomic $(2 \times 2)$ configurations provides sufficient results to allow for interesting deductions concerning the higher-order reconstructions.

The entire subject will be discussed in two parts. This paper (paper I) will describe the general methodology of studying polar semiconductor surfaces and its application to the reconstructions of the (111) surface of GaAs. The following paper (paper II) is concerned with the reconstructions and related phase transitions of the $(\overline{1} \overline{1} \overline{1})$ surface of GaAs. The format of the present paper is as follows: Section II contains a description of the theoretical model for the surface and the computational scheme for the total energy. Section III discusses the geometric features of the different reconstruction models for the (111) surface, and the total energy of each model. Section 
IV introduces the definition of the relative chemical potential of $\mathrm{Ga}$ and As atoms in the context of the calculations, and discusses its effect on the total energies. Finally, Sec. V makes contact with experiment using the information from the total energies and the chemical potentials and draws conclusions on the appropriate reconstruction models for particular atomic environments (As- or Garich).

\section{SURFACE MODEL AND COMPUTATIONAL SCHEME}

The surface is modeled by a slab geometry contained by vacuum regions and periodically repeated in the direction perpendicular to the surface. This supercell configuration introduces the first major complication: a slab of GaAs cut along the [111] direction exposes a different surface on each side, namely the (111) and $(\overline{1} \overline{1} \overline{1})$ surfaces. In a repeated configuration the different polarities of the two surfaces will introduce spurious fields in the vacuum region, which will strongly affect the surface reconstructions. This is shown schematically in Fig. 1(a): the average potential increases from one surface to the other
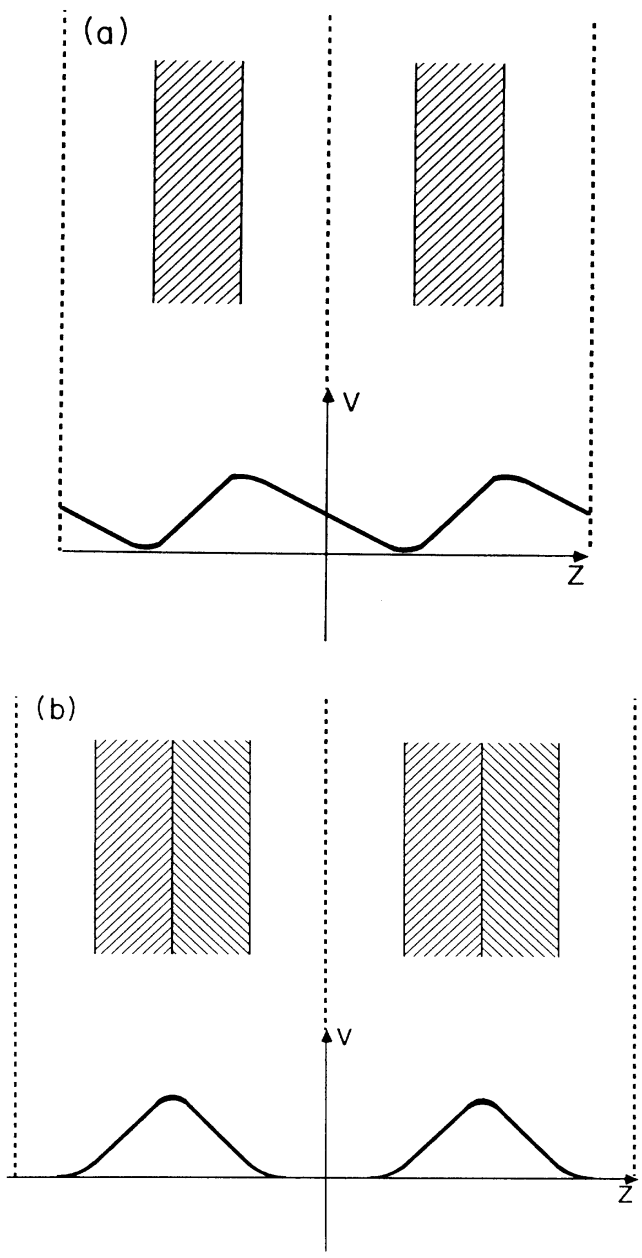

FIG. 1. Average potential in supercell configuration: (a) slabs without inversion symmetry; (b) slabs with inversion symmetry. Dashed lines indicate supercell boundaries. through the slab and the periodicity introduces variations of the potential in the vacuum. To solve this problem we considered slabs with artificially-imposed inversion symmetry. This is shown in Fig. 1(b) and illustrates how the spurious vacuum fields are canceled. The center of the slab contains two planes of the same kind of atoms. This region was frozen throughout the calculations.

A single slab would, artificially, give rise to charge transfer from one surface to the other. This is a consequence of the inequivalent dangling-bond states associated with the two surfaces: partially filled states associated with the As-terminated surface would be lower in energy than the corresponding states of the Ga-terminated surface. A self-consistent iterative approach will initially place some excess charge in the low-lying states producing a charge buildup which in turn will raise the energy of these states. The ensuing charge "sloshing" is completely unphysical and prohibits convergence.

The double slab successfully solves this problem as well, albeit with some minor modifications of the atomic potentials. The inversion symmetry leads to a charge buildup at the interface of the two same-atom planes. The two center planes were therefore modeled by partially charged fictitious atoms which are bonded together to avoid charge transfer from the interface to the surface. Fictitious As atoms appear at the center of the slab with the (111) surface exposed, and similarly, fictitious Ga atoms appear at the center of the slab with the $(\overline{1} \overline{1} \overline{1})$ surface exposed. These ideas can be further elucidated using a simple tight-binding argument. For a slab with the (111) surface exposed, two $(\overline{1} \overline{1} \overline{1})$ surface are brought together at the interface and the dangling bonds in this region contain $\frac{5}{4} e^{-}$each since they are As- $s p^{3}$ orbitals. The interaction of these orbitals creates a bonding and an antibonding state, with the bonding state completely filled and the antibonding one containing the excess $\frac{1}{2} e^{-}$. However, the Ga dangling bonds of the slab surface lie lower in energy than the antibonding state of the interface region, and they are partially filled Ga- $s p^{3}$ orbitals, containing $\frac{3}{4} e^{-}$ each. This will lead to artificial charge transfer from the high-energy antibonding interface states to the lowerenergy dangling-bond surface states. By making the interface As atoms have valence charge 4.75 instead of 5 , the excess charge is removed from the interface region and the artificial charge transfer is avoided. Similar arguments indicate that the interface fictitious $\mathrm{Ga}$ atoms should have valence charge 3.25 instead of 3 .

The above approximation will not affect the surface reconstruction energies, as long as the interface is sufficiently far from the surface. In our model there are eight atomic planes between the interface region and the surface (the usual thickness of slabs for similar calculations), resulting in a slab of sixteen atomic layers. The vacuum region between the slabs is equivalent to four GaAs bond lengths.

The atoms were modeled by nonlocal norm-conserving pseudopotentials constructed by the method of Hamman, Schlüter, and Chiang, ${ }^{10}$ which are shown in Figs. $2(a)$ and 2(b) for $\mathrm{Ga}$ and As, respectively. In the same figures the potentials of the corresponding fictitious interface atoms are shown by dashed lines. It is obvious that the latter po- 
tentials are very similar to the original $\mathrm{Ga}$ and As potentials, except for the different valence charge, reflected in a small overall shift.

The total energy of each atomic configuration was calculated using the self-consistent local-density-functional formalism, ${ }^{11}$ with the Wigner approximation ${ }^{12}$ for the exchange-correlation energy. The calculations in the momentum-space representation ${ }^{13}$ involve a basis of plane waves with energy up to $E_{1}=4 \mathrm{Ry}$ ( $\sim 1700$ plane waves). An additional set of plane waves with energy up to $E_{2}=8$
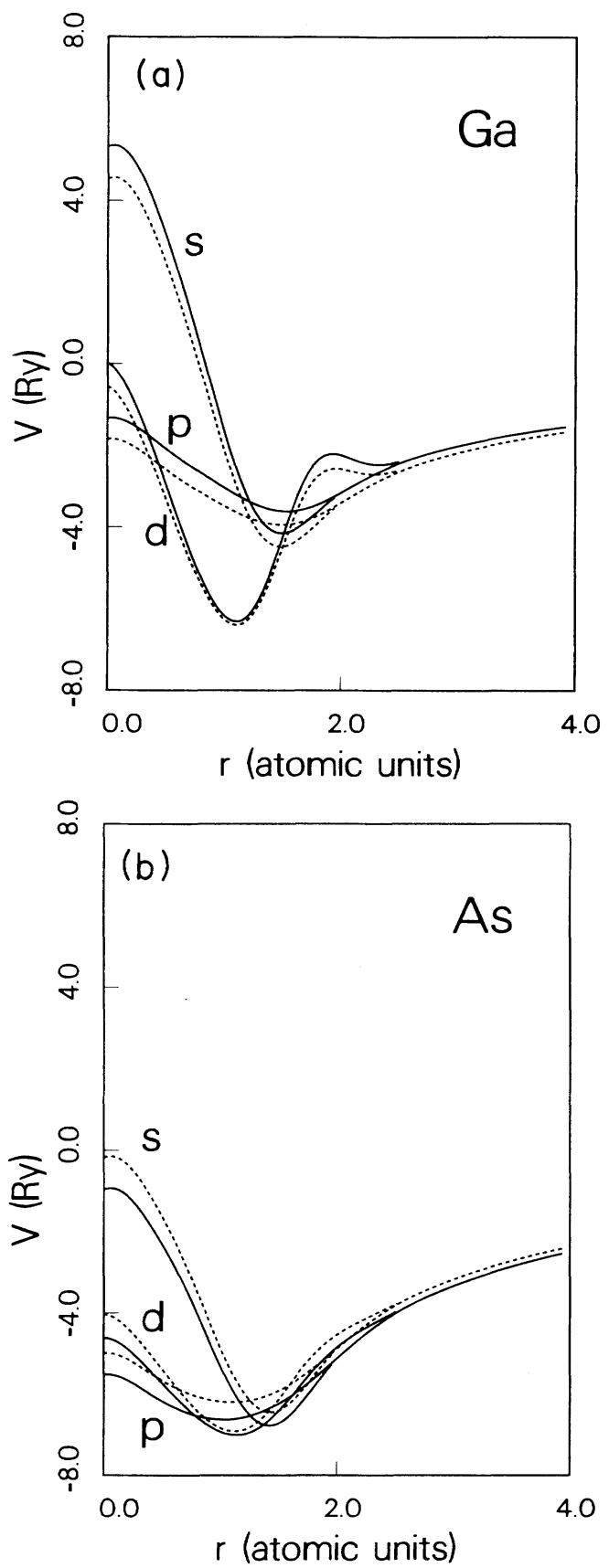

FIG. 2. Nonlocal pseudopotentials: (a) Ga (solid lines) and fictitious Ga (dashed lines). (b) As (solid lines) and fictitious As (dashed lines).
Ry ( $\sim 3500$ plane waves) is included through second-order perturbation theory. ${ }^{14}$ It has been established before ${ }^{15}$ that such basis set cutoffs are sufficient for accurate surface calculations in problems involving Ga and As atoms. Four points in the surface $\left(k_{z}=0\right)$ Brillouin zone were used to calculate the wave functions and band energies. These were chosen by the Monkhorst-Pack method ${ }^{16}$ and were shifted to high-symmetry points to facilitate the calculation. For each reconstruction model extensive relaxation was allowed by minimizing the energy with respect to the most important surface relaxation parameters.

In order to compare the energies of the different reconstruction models, a consistent way to incorporate the energy of single atoms is needed. The pseudopotential approximation gives a straightforward evaluation of the energy of isolated atoms through real-space integration of the kinetic, Coulomb, and exchange correlation energies, in the local-density-functional formalism. However, it is not realistic to include the energies of isolated atoms in the comparison of surfaces with different numbers of atoms. A more appropriate comparison is to subtract from the atomic energies the binding energy of atomic reservoirs where the atoms might end up when removed from the surface, or, equivalently, where the atoms are taken from to be added to the surface. One obvious choice of atomic reservoir is GaAs bulk: breaking bulk pairs gives one $\mathrm{Ga}$ and one As atom available to participate in surface reconstructions; conversely, pairs of Ga and As atoms removed from the surface can be considered to become part of the bulk-by extending a surface step for instance, which in the thermodynamic limit gives an equivalent configuration. This reservoir choice is unambiguous assuming that the surface is in equilibrium with the bulk. It is less obvious what the proper reservoir choices are for excess $\mathrm{Ga}$ or As atoms, when they cannot be paired to GaAs. The usual experimental setup for MBE growth $^{1,6}$ of the surfaces considered here suggests that two possible choices are $\mathrm{Ga}$ bulk and $\mathrm{As}_{2}$ gas. These two reservoirs will be used throughout the present work as sources or sinks of single atoms of each kind. $\mathrm{As}_{4}$ gas will also be considered as an As-reservoir choice, since it is the most stable gaseous form of As.

The above definition of the atomic energies relative to the surface is in effect an approximation of the chemical potential of the atoms. The usual definition of the chemical potential is $\mu=(\partial E / \partial N)$, where $E$ is the total energy and $N$ the number of atoms in the system. Defining $\varepsilon_{a}$ to be the energy of an isolated atom, and $\varepsilon_{b}$ the binding energy per atom for a specific reservoir, we can write the total energy as $E=N \varepsilon_{a}-N \varepsilon_{b}$, which gives for the chemical potential $\mu=\varepsilon_{a}-\varepsilon_{b}-N \partial \varepsilon_{b} / \partial N$. In the case of a solid atomic reservoir, the last term in this equation should vanish in the thermodynamic limit. In the case of a gas reservoir the same term can be written as $\rho \partial \varepsilon_{b} / \partial \rho$, where $\rho=N / V$ is the density of the system and we have assumed that the volume is constant. The binding energy $\varepsilon_{b}$ in a molecular gas $\left(\mathrm{As}_{2}\right.$ or $\mathrm{As}_{4}$, for example) is due to the interaction between atoms in a molecule (which is density independent) and the interaction between molecules (which gives rise to the density dependence). The interaction between molecules is much weaker than that of atoms 
in a molecule so this part of $\varepsilon_{b}$ as well as well as the density dependence can be ignored, giving $\mu=\varepsilon_{a}-\varepsilon_{b}$, as described in the previous paragraph.

The effect of considering different reservoirs as sources and sinks will be discussed in Sec. V. The binding energies for the reservoirs were taken from experiment: ${ }^{17} 2.8$ $\mathrm{eV}$ per atom for $\mathrm{Ga}$ bulk, $2.0 \mathrm{eV}$ per atom for $\mathrm{As}_{2}$ gas, and $2.7 \mathrm{eV}$ per atom for $\mathrm{As}_{4}$ gas. The binding energy of bulk GaAs was calculated to be $6.8 \mathrm{eV}$ per GaAs pair (the experimental value is $6.7 \mathrm{eV}$ ).

Another important feature of the energy comparisons is the classification of the different reconstruction models in stoichiometric classes. For the purposes of this work, a convenient definition of stoichiometry is $S=\left(N_{\mathrm{As}}\right.$ $\left.-N_{\mathrm{Ga}}\right) / 4$, where $N_{\mathrm{As}}\left(N_{\mathrm{Ga}}\right)$ is the number of As $(\mathrm{Ga})$ atoms in the surface bilayer of the $(2 \times 2)$ unit cell. Thus $\Delta S=1$ between two surfaces indicates a difference equivalent to a whole monolayer of atoms of one kind. The above definition of $S$ will prove helpful in determining the metallic or semiconducting character of the different model geometries and in discussing the phase transitions between different reconstructions (see paper II). It will also be useful in describing the dependence of the total energy on the relative chemical potential (see Sec. V).

It is important to point out that the ideal unreconstructed surface has metallic character due to the number of broken bonds per $(2 \times 2)$ unit cell and their effective charge content, namely $\frac{3}{4} e^{-}$for $\mathrm{Ga}$ broken bonds and $\frac{5}{4} e^{-}$for As broken bonds. Only surfaces differing from the ideal by $\Delta S=(n / 4)$ where $n$ is an odd integer can have semiconducting character; this is a necessary but not sufficient condition since the semiconducting character must also be reflected by a band-structure gap. The ideal unreconstructed surface has $S=0$, and the calculated total energy of this configuration will also be taken to define the zero of energy.

Having established a consistent way to to compare the total energy of surfaces with different numbers of atoms, and a classification scheme according to the relative As and Ga content of the surface bilayer, we can now search for appropriate reconstruction models. In this search we will follow the guidelines that threefold-coordinated $\mathrm{Ga}$ atoms tend to assume a planar $s p^{2}$ bonding configuration while threefold coordinated As atoms assume a pyramidlike $p^{3}$ bonding configuration. This idea is based on the atomic properties of the elements; $\mathrm{Ga}$ has three valence electrons which for a single atom lead to three $s p^{2}$ bonding orbitals, while As has five valence electrons which tend to form a filled $s^{2}$ atomic state and three $p^{3}$ bonding orbitals. The expectation that these configurations produce low-energy reconstructions is borne out by our calculations and has been crucial in determining the relaxation of the (110) surface of GaAs. This surface is nonpolar, containing one $\mathrm{Ga}$ and one As atom per surface unit cell, which in their relaxed positions assume exactly the configurations described above, as has been confirmed both experimentally ${ }^{18}$ and theoretically. ${ }^{19}$ The near perfect atomic relaxation is achieved by simply tilting the As atoms upwards and the Ga atoms downwards. These ideas are not directly applicable to the reconstruction of the [111] surfaces: the surface atoms are at the same time threefold coordinated and constrained by their neighbors to have an $s p^{3}$ bonding configuration, with one partiallyfilled bonding orbital directed vertically out of the surface (the so-called dangling bond). There is no simple way for all the surface atoms to achieve a favorable coordination and rid themselves of the energetically costly dangling bonds. With these general remarks in mind we will discuss the possible reconstructions that lower the surface energy by eliminating the dangling bonds and reduce the polar character.

\section{III. (111) SURFACE RECONSTRUCTIONS}

Perspective views of the structural models for this surface are shown in Fig. 3; Fig. 3(a) is the ideal unreconstructed surface. In order to establish whether a particular geometry has semiconducting character, a schematic band structure is given in Fig. 4, for those models that have $S=(n / 4), n=o d d$. This band structure is based on a linear interpolation of the energy between the calculated values at the $\Gamma$ and $M$ points of the surface Brillouin zone. It is intended as a rough lower bound of the gap for the different atomic configurations, given the inherent difficulties of the density-functional theory in calculating the gap. ${ }^{20}$ In general, it is expected that the most stable reconstructions will have semiconducting character and the corresponding models will be favored, although this is by no means an absolute criterion for stability. The schematic band structure of the ideal surface is included in Fig. 4 for purposes of comparison. The surface dangling bonds form a fourfold degenerate band which is intersected by the Fermi level.

The total energies [in eV per $(2 \times 2)$ unit cell] with respect to the ideal surface, and the deviations from stoichiometry of the different reconstructions are given in Table I.

The simplest $(2 \times 2)$ reconstruction of the ideal surface consists of buckling ${ }^{21}$ in which one out of four surface $\mathrm{Ga}$ atoms moves outward from the surface while the other three surface Ga atoms move inward [Fig. 3(b)]. This allows for partial elimination of the surface dangling bonds on the three inward relaxed $\mathrm{Ga}$ atoms which become roughly coplanar with their As nearest neighbors. The outward moving $\mathrm{Ga}$ atom forms three bonds which are stretched by $8 \%$ with respect to the ideal bond length. Owing to rehybridization, an $s$-like state associated with this atom is filled and the stretched bonds are weakened. The necessary amount of electronic charge to fill these bonds is provided by partial charge transfer from the three dangling bonds of the planar Ga atoms. The relaxation brings the $\mathrm{Ga}$ and As atoms of the surface bilayer (except for the upward buckled $\mathrm{Ga}$ atom) almost to the same plane, thereby reducing the polarity of the surface. The energy of the buckled configuration is $0.8 \mathrm{eV}$ lower than the ideal surface and its stoichiometry is $S=0$, indicating a metallic surface.

Since the coordination of the $\mathrm{Ga}$ atom at the buckled position is unfavorable, it is interesting to explore the possibility of an As atom replacing it. This leads to the sub- 

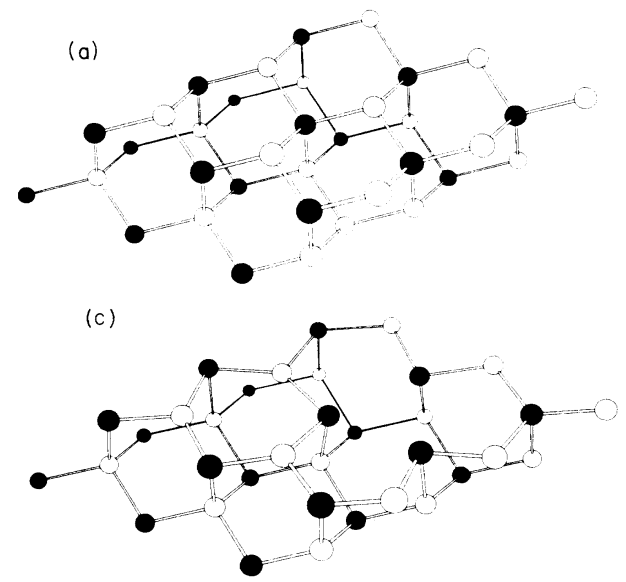
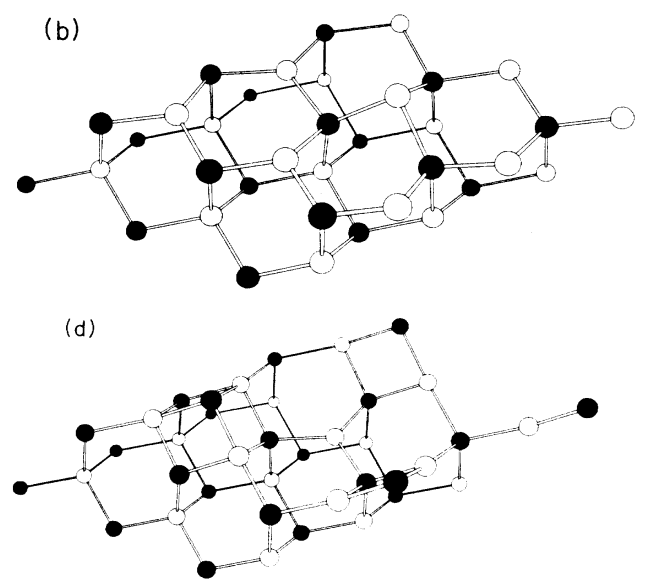

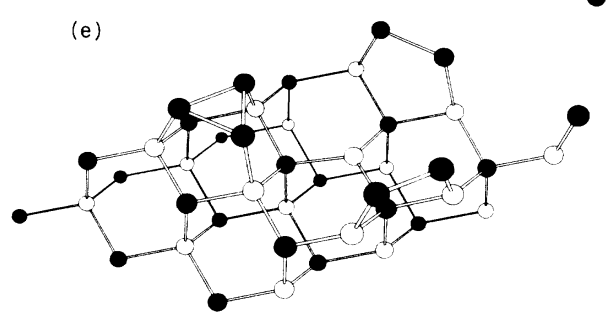

FIG. 3. Structural models for the (111) surface $(2 \times 2)$ reconstructions. The first four atomic layers are shown in perspective. The atoms in each layer outline the $(2 \times 2)$ unit cell, in the ideal configuration. The same atoms and their nearest neighbors are shown in the other reconstructions. Solid circles represent As atoms and empty circles represent Ga atoms. (a) Ideal surface. (b) Buckled geometry; a variation of this is the substitutional geometry. (c) Vacancy geometry; variations of this are the Ga-vacancy plus Gasubstitutional geometry and the Ga-vacancy plus As p-bonded geometry. (d) Adatom geometry. (e) Triangle geometry.

stitutional configuration ${ }^{22}$ in which the As atom is $p^{3}$ bonded to three subsurface As atoms. There is a tradeoff between the energy gain from the favorable relaxation of the As atom and the formation of three As-As bonds which are higher in energy than the Ga-As bonds. After allowing for relaxation we find that the energy of the substitutional model is equal to the buckled geometry in Asrich environment (assuming that the removed $\mathrm{Ga}$ atom combines with excess As from the $\mathrm{As}_{2}$ reservoir to form a GaAs bulk pair). Thus the substitution is neither favored nor inhibited energetically over the buckling. The substi- tutional model has also metallic character $\left(S=\frac{2}{4}\right)$.

If the buckled $\mathrm{Ga}$ atom is removed vertically out of the surface, the energy increases monotonically as bonds are being broken until the atom no longer interacts with the surface (at about $5 \mathrm{~A}$ away). The energy versus the vertical position of the removed $\mathrm{Ga}$ atom is shown in Fig. 5 . The minimum of the energy curve corresponds to the buckled geometry. The valence charge density for four different configurations during the removal of the Ga atom is shown in Fig. 6. The plane of this figure is a (110) plane along the long diagonal of the unit cell. The

TABLE I. Total energies and stoichiometries for the different $(2 \times 2)$ reconstruction models of the (111) surface. The energies are given in $\mathrm{eV}$ per $(2 \times 2)$ unit cell with respect to the ideal unreconstructed surface. The different models are arranged in stoichiometric classes.

\begin{tabular}{lcr}
\hline \hline \multicolumn{1}{c}{ Model geometry } & Stoichiometry & Energy \\
\hline (1) Ga vacancy + Ga substitutional & $-\frac{1}{4}$ & -0.3 \\
(2.) Buckled surface & 0 & -0.8 \\
(3) Ga vacancy & $\frac{1}{4}$ & -3.3 \\
(4) Ga vacancy + As p bonded & $\frac{1}{4}$ & -1.9 \\
(5) As adatom & $\frac{1}{4}$ & -0.8 \\
(6) As substitutional & $\frac{2}{4}$ & -0.8 \\
(7) Ga vacancy + As adatom & $\frac{2}{4}$ & 0.0 \\
(8) As triangle & $\frac{3}{4}$ & -5.0 \\
\hline \hline
\end{tabular}



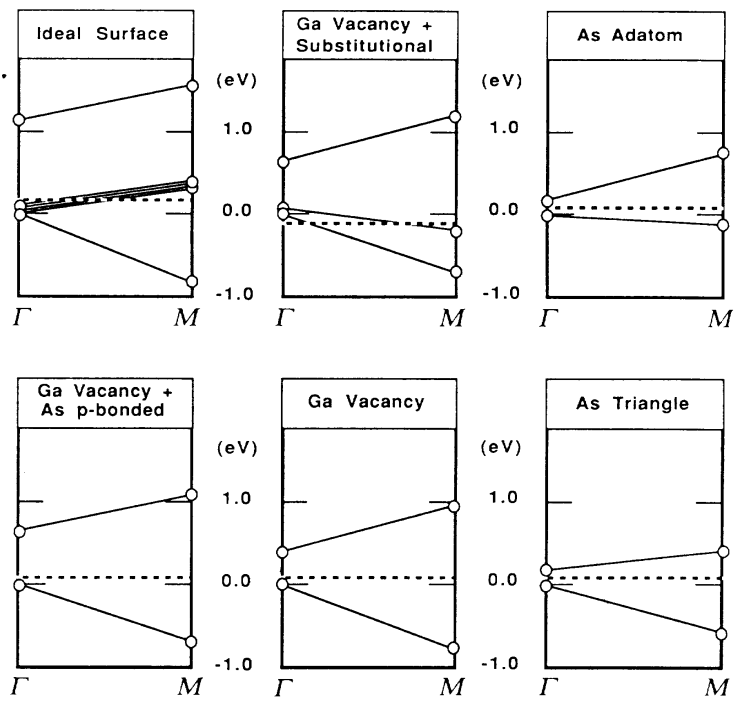

FIG. 4. Schematic band structure for the different surface reconstructions with $S=n / 4, n=o d d$, and the ideal surface. The dashed line is the Fermi level, placed at the highest filled band. The dots are the calculated energies at the $\Gamma$ and $M$ points of the surface Brillouin zone.

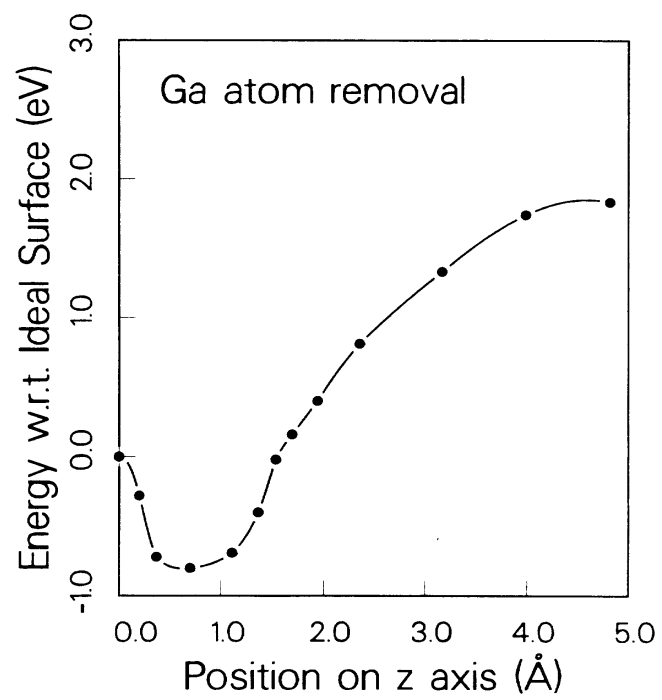

FIG. 5. Energy versus vertical position of the removed Ga atom from the (111) ideal surface; the minimum represents the buckled configuration.
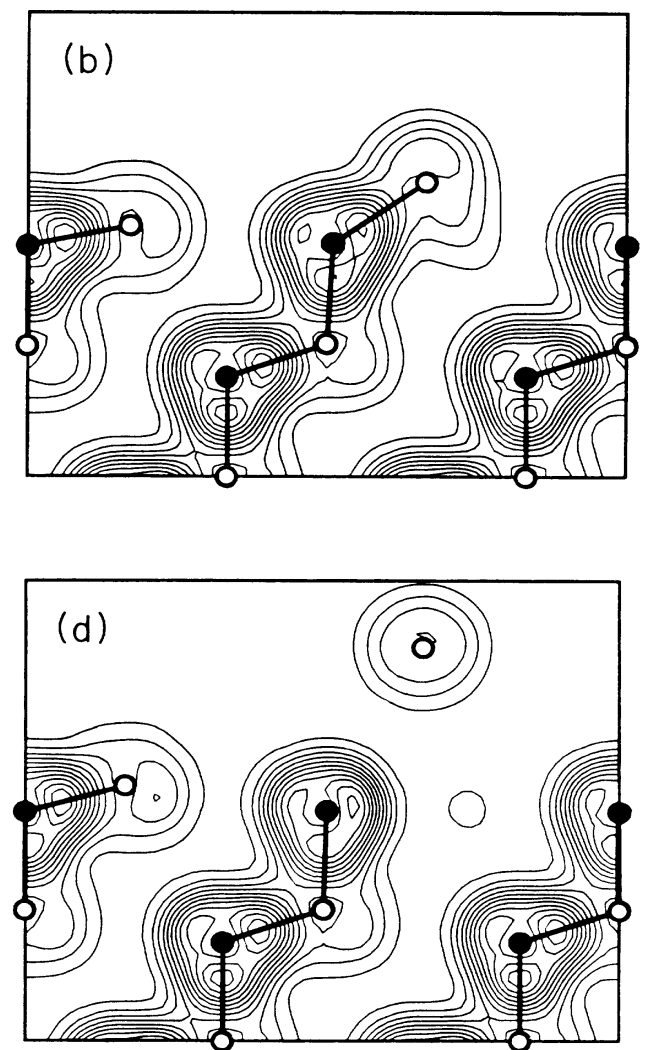

FIG. 6. Charge density on the (110) plane for four configurations during the removal of a Ga atom from the (111) surface: (a) ideal surface; (b) buckled geometry; (c) Ga atom at $\Delta z=1.7 \AA$ from ideal position; (d) Ga atom at $\Delta z=3.2 \AA$ from ideal position. 


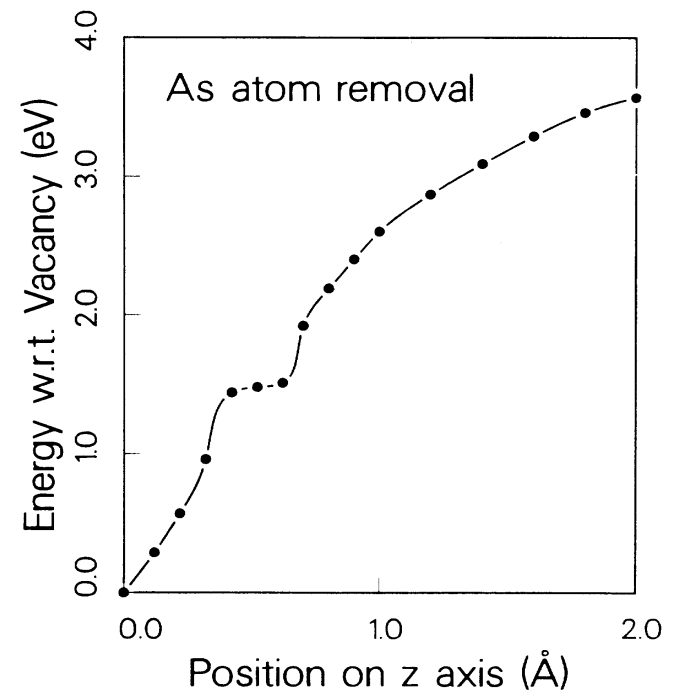

FIG. 7. Energy versus vertical position of the removed As atom from the (111) Ga-vacancy geometry; the step represents the most favorable local binding arrangement of the Ga vacancy plus As $p$-bonded configuration.

atoms are represented by solid (As) or empty circles $(\mathrm{Ga})$. Figure 6(a) corresponds to the ideal unreconstructed surface; Fig. 6(b) corresponds to the buckled geometry; Fig. 6(c) shows the Ga atom at $1.7 \AA$ away from its ideal position, with the bonds already broken; finally, in Fig. 6(d) the $\mathrm{Ga}$ atom at $3.2 \AA$ away is no longer bonded to the surface, although there is some residual interaction, as can be inferred from the total-energy curve of Fig. 5.

When the buckled $\mathrm{Ga}$ atom has been completely removed from the surface, a vacancy configuration of particular stability is reached [Fig. 3(c)]. The stability is a result of the favorable relaxation of the remaining surface atoms: the three As atoms surrounding the vacancy site relax toward this site and attain an almost $p^{3}$ bonding configuration; the surface $\mathrm{Ga}$ atoms have an $s p^{2}$ bonding configuration, coplanar with their neighboring As atoms and significantly better than in the buckled geometry, due to the simultaneous relaxation of the As atoms. This geometry, proposed by Tong, $\mathrm{Xu}$, and $\mathrm{Mei}^{4}$ and by $\mathrm{Chadi}^{7}$ is a serious contender for the most stable surface reconstruction. Its energy is $3.3 \mathrm{eV}$ lower than the ideal surface, assuming that the removed $\mathrm{Ga}$ atom combines with excess As from the $\mathrm{As}_{2}$ reservoir to form a GaAs bulk pair. It is also important to notice that the vacancy geometry has stoichiometry $S=\frac{1}{4}$ and has semiconducting character with a gap of $\sim 0.44 \mathrm{eV}$ (see Fig. 4).

In the vacancy geometry, the single surface atom with unfavorable coordination is the As atom of the surface bilayer which is not adjacent to the vacancy site. It has three $\mathrm{Ga}$ neighbors on the same plane and a fourth $\mathrm{Ga}$ neighbor directly below it. Thus three of its bonds lie on a plane and the fourth is perpendicular to this plane. We have explored the possibility of moving this As atom to a more favorable position: it is placed at a site above the plane of the three Ga atoms, assuming a $p^{3}$ bonding configuration, while its bond with the fourth $\mathrm{Ga}$ atom is completely broken. This does not destroy the favorable relaxation of the $\mathrm{Ga}$ atoms since they still remain $s p^{2}$ bonded, although their bonds lie on planes tilted with respect to the surface plane. The $\mathrm{Ga}$ atom on the second bilayer with one bond broken is also allowed to relax by a small amount downward. This configuration maintains the semiconducting character of the vacancy geometry, having $S=\frac{1}{4}$ and a gap of $\sim 0.63 \mathrm{eV}$ (see Fig. 4). The relaxation of the displaced As atom however, is not sufficient to compensate for the energy cost of breaking one of the bonds. The total energy of this model is $1.4 \mathrm{eV}$ higher than that of the $\mathrm{Ga}$ vacancy, at $-1.9 \mathrm{eV}$ with respect to the ideal surface. The energy versus the position of the displaced As atom is given in Fig. 7. The most favorable local binding position is indicated by the step in the energy curve. At this point the As atom has attained its maximum relaxation; further displacement leads to breaking of its bonds, accompanied by a continuous increase in the energy. In Fig. 8 we show the valence charge density of the Ga-vacancy configuration [Fig. 8(a)] and the Ga vacancy plus As $p$-bonded configuration [Fig. 8(b)]. From the charge densities it is evident that the bond of the $p$ bonded As atom to the $\mathrm{Ga}$ atom directly below it is broken, whereas the rest of the bonds are not severely distorted.

Another possibility is the replacement of the unfavorably coordinated surface As atom by a $\mathrm{Ga}$ atom. This amounts to effectively removing only one As atom from the surface. The resulting configuration is the Ga vacan-
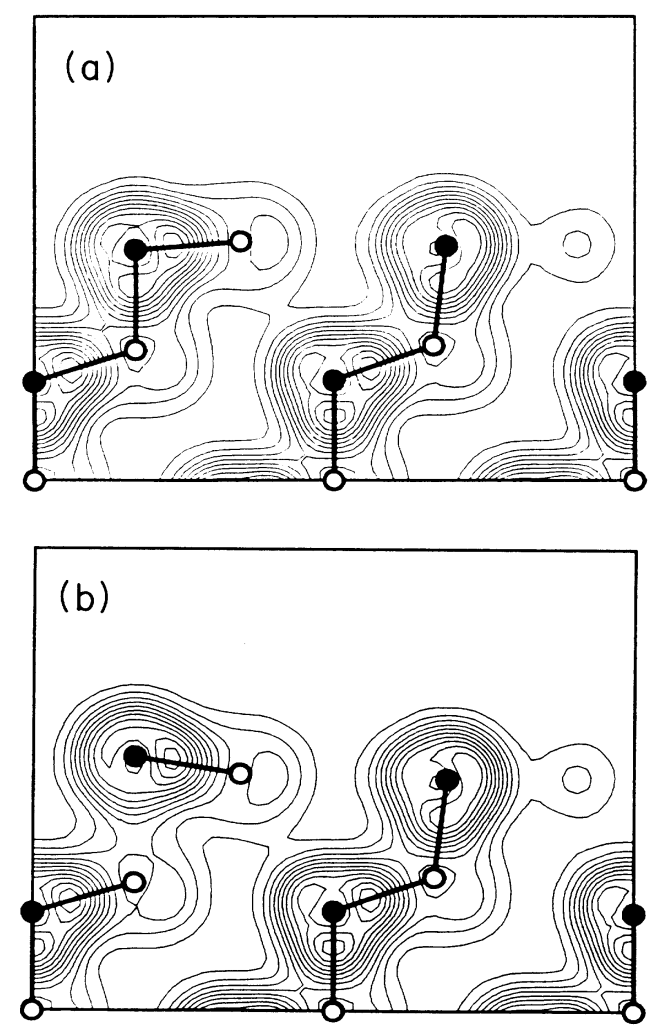

FIG. 8. Charge density on the (110) plane for two vacancy reconstructions of the (111) surface: (a) Ga vacancy; (b) Ga vacancy plus As $p$ bonded. 
cy plus Ga substitutional. Assuming that the removed As atom combines with excess $\mathrm{Ga}$ from $\mathrm{Ga}$ bulk to form a GaAs pair, we find that the energy of this model is -0.3 $\mathrm{eV}$ with respect to the ideal surface. This relatively high energy can be traced to the four $\mathrm{Ga}-\mathrm{Ga}$ bonds due to the substitution of an as atom by a Ga atom. This substitution introduces a deficiency in electronic charge which results in a metallic system (see Fig. 4) even though the stoichiometry satisfies the condition for semiconducting character $\left(S=-\frac{1}{4}\right)$.

A different class of models for the $(2 \times 2)$ reconstructions involves the addition of atoms to the ideal surface. Thus by adding one As atom bonded to three Ga atoms in the $(2 \times 2)$ surface unit cell, three of the Ga dangling bonds are eliminated. The fourth surface $\mathrm{Ga}$ atom is allowed to relax downward and becomes coplanar with its As nearest neighbors [Fig. 3(d)]. This relaxation leads to rehybridization and partial charge transfer in the opposite sense of the $\mathrm{Ga}$ vacancy. The resulting adatom geometry $^{23}$ has stoichiometry $S=\frac{1}{4}$ and a rather small energy gap of $\sim 0.14 \mathrm{eV}$. Its energy lies at $-0.8 \mathrm{eV}$ below the ideal surface plus excess $\mathrm{As}_{2}$ gas.

It is interesting to ask whether the As adatom combined with the already low-energy vacancy geometry would give a still lower-energy configuration. The combined model however has an energy $0.0 \mathrm{eV}$ with respect to the ideal surface plus excess $\mathrm{As}_{2}$ gas, after allowing for the removed $\mathrm{Ga}$ atom to form a GaAs bulk pair with the available As. The reason for this high energy relative to the vacancy and adatom models, is the conflicting nature of the relaxations involved in these two configurations. The vacancy relaxation is similar to the buckled geometry in which one out of four surface Ga atoms moves outward and the other three move inward to become planar with the subsurface As atoms. In the adatom relaxation the displacement of the surface $\mathrm{Ga}$ atoms is opposite in order to accomodate the added As atom. This conflict eliminates all the energy gain from the relaxations and results in an energetically unfavorable configuration. The Gavacancy plus As-adatom geometry has metallic character with stoichiometry $S=\frac{2}{4}$.

A closer examination of the adatom geometry reveals that the bonding of the As atom is not ideal: it forms three four-atom rings with high local charge density which should be costly in energy. At the same time the bond between a surface Ga atom and the As adatom is tilted by a large angle with respect to the vertical which would be its preferred direction, dictated by the other three $s p^{3}$ bonds of the Ga atom. These arguments led to the search for a more appropriate adatom model. An interesting candidate proved to be the triangle geometry. ${ }^{24}$ In this geometry, three As adatoms are bonded to three surface $\mathrm{Ga}$ atoms and to each other in an equilateral triangle configuration. The fourth surface $\mathrm{Ga}$ atom is then relaxed in the same manner as in the single adatom geometry [Fig. 3(e)]. The stoichiometry of this reconstruction is $S=\frac{3}{4}$ and it has semiconducting character with a gap of $\sim 0.16 \mathrm{eV}$ (Fig. 4). Its energy is $5.0 \mathrm{eV}$ lower than the ideal surface plus excess $\mathrm{As}_{2}$ gas. This is by far the lowest-energy configuration, a full $1.7 \mathrm{eV}$ lower than the Ga vacancy. Two reasons contribute to this low energy. First, the bonding of the As atoms in the triangle gives a lowering of the energy per adatom roughly equal to the binding energy per atom in the $\mathrm{As}_{2}$ reservoir; thus not much energy is wasted in removing single atoms from the reservoir in order to deposit them on the surface. Second, the triangular arrangement of the As adatoms allows them to form almost vertical bonds with the surface $\mathrm{Ga}$ atoms; as a result the surface charge density is not severely distorted. Figure 9 presents the valence-charge densities of the As adatom [Fig. 9(a)] and As-triangle geometries [Fig. 9(b)]. In the As-triangle model the bonds of the adatoms to the surface are very similar in nature to the bulk GaAs bonds, whereas in the As adatom model there are large deviations from the bulk-bonding charge distribution. Note also the weakening of the back bonds of the first bilayer As atoms in the As adatom configuration relative to the bulk bonds.

Judging from energy considerations alone, the triangle geometry appears to be ideally suited for an As-rich surface reconstruction model. Its realization however might be hindered by kinematic effects and its range of stability might be narrow due to the high As content (as will be discussed in connection with the chemical potentials). Nevertheless, it could be particularly important in nonequilibrium situations, such as crystal growth: the very low energy indicates that if enough As can be supplied to the surface the As triangles should be readily formed.
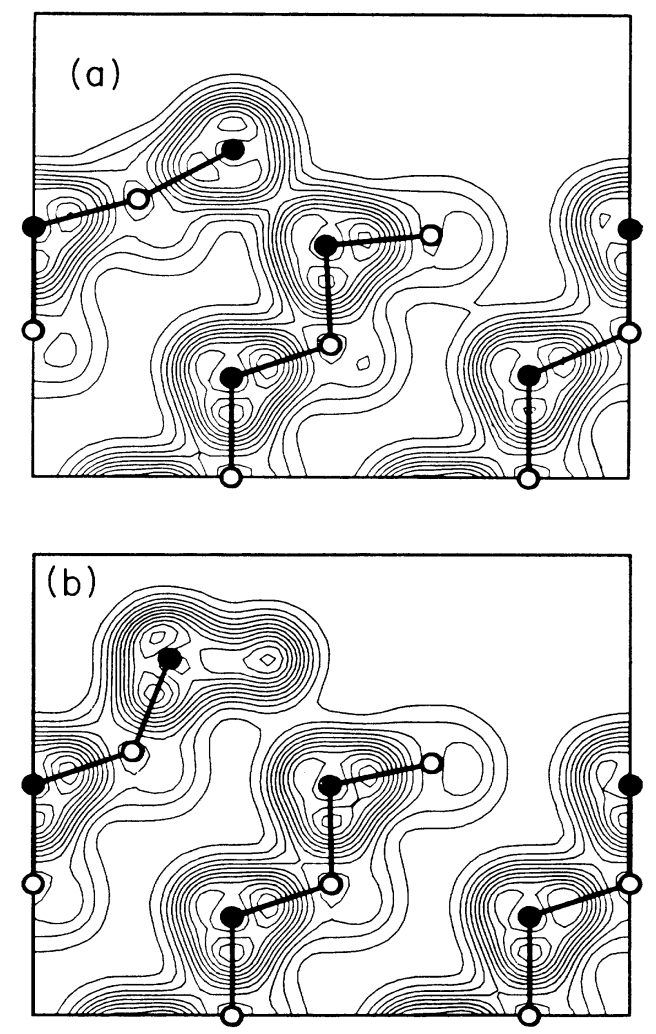

FIG. 9. Charge density on the (110) plane for two adatom reconstructions of the (111) surface: (a) As adatom; (b) As triangle. 


\section{EFFECT OF RELATIVE CHEMICAL POTENTIAL}

Up to this point the energies for the different reconstructions have been calculated by assuming that each surface is in equilibrium with its natural environment and with bulk GaAs. Specifically, an As-rich surface (having higher As content than the ideal by addition of As atoms or removal of $\mathrm{Ga}$ atoms) is in equilibrium with $\mathrm{As}_{2}$ gas and the removed $\mathrm{Ga}$ atoms are paired with As atoms to give GaAs bulk. Similarly, a Ga-rich surface is in equilibrium with $\mathrm{Ga}$ bulk and the removed As atoms are paired with $\mathrm{Ga}$ atoms to GaAs bulk. This however need not be the case as far as preparation conditions for the surface are concerned. For example, an As-rich surface might be prepared with excess $\mathrm{Ga}$ present. In this case if the Asrich character of the surface is due to removal of $\mathrm{Ga}$ atoms, these $\mathrm{Ga}$ atoms will necessarily be incorporated in Ga bulk; conversely, if the As-rich character is due to addition of As atoms these As atoms will have to be obtained from beaking GaAs pairs, whereupon the remaining unpaired $\mathrm{Ga}$ atoms will be added to $\mathrm{Ga}$ bulk. Since the binding energy per atom is $2.0 \mathrm{eV}$ for $\mathrm{As}_{2}$ gas and 2.8 $\mathrm{eV}$ per atom for $\mathrm{Ga}$ bulk, and the cohesive energy of $\mathrm{GaAs}$ is $6.8 \mathrm{eV}$ per pair, it is readily seen that by changing from $\mathrm{As}_{2}$ environment to $\mathrm{Ga}$ bulk environment, the energy cost is $n(2.0 \mathrm{eV})$ for a stoichiometric change of $\Delta S=n / 4$, on an As-rich $(S>0)$ surface. Similar considerations hold for Ga-rich surfaces. There is however a range of intermediate environments between the As- and Ga-saturated environments. Accordingly, the energy cost for these intermediate environments will be a fraction of the above-mentioned $2.0-\mathrm{eV}$ range. We denote this relative chemical potential between the $\mathrm{Ga}$ and As atoms by $\delta \mu$. The preceding discussion showed that the range of $\delta \mu$ is $2.0 \mathrm{eV}$, and since the zero of the relative chemical potential is arbitrary, we define $\delta \mu=1.0 \mathrm{eV}$ to be the Assaturated environment and $\delta \mu=-1.0 \mathrm{eV}$ to be the $\mathrm{Ga}-$ saturated environment. Using the definition of stoichiometry $S$ we can then write the following equations for the energies of As- and Ga-rich surfaces in arbitrary environment which is defined by the value of $\delta \mu$ :

$$
\begin{aligned}
& E_{\mathrm{As}-\text { rich }}(\delta \mu)=E_{\mathrm{As}-\text { rich }}^{\mathrm{eq}}+4 S(1.0 \mathrm{eV}-\delta \mu), \\
& E_{\mathrm{Ga}-\text { rich }}(\delta \mu)=E_{\mathrm{Ga} \text {-rich }}^{\mathrm{eq} .}-4 S(1.0 \mathrm{eV}+\delta \mu) .
\end{aligned}
$$

where $E_{\mathrm{As} \text {-rich }}^{\text {eq. }}$ and $E_{\mathrm{Ga-rich}}^{\text {eq. }}$ are the total energies of the As-rich $(S>0)$ and Ga-rich $(S<0)$ surfaces in equilibrium with $\mathrm{As}_{2}$ and $\mathrm{Ga}$ bulk environments as given in Table I.

These considerations give a more complete picture of the surface reconstruction energies under all possible environments, which is depicted graphically in Fig. 10. From this figure alone we can predict that phase transitions will occur as the relative chemical potential $\delta \mu$ scans its range, whenever two lowest-energy lines cross and different reconstructions become the lowest-energy configuration. One such crossing occurs in Fig. 10. At this point however it is important to recall that our search was restricted to a subset of $(2 \times 2)$ reconstructions (the large number of models examined does not necessarily exhaust all the possibilities). There might actually exist more complicated

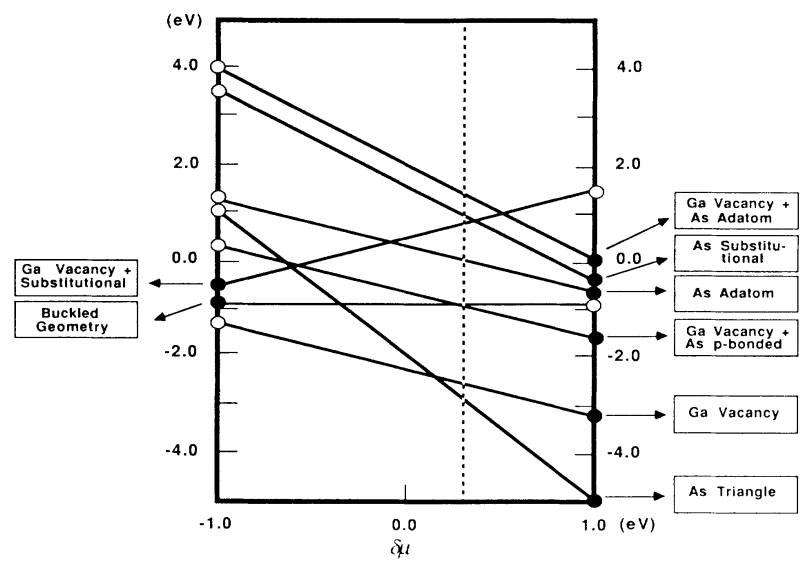

FIG. 10. Energy versus relative chemical potential $\delta \mu$ of the different reconstruction models of the (111) surface. The range of $\delta \mu$ scans the values consistent with the Ga bulk and $\mathrm{As}_{2}$ gas reservoirs. The solid dots are the calculated energies per $(2 \times 2)$ unit cell with respect to the ideal surface. The dashed line indicates the upper limit of $\delta \mu$ consistent with an $\mathrm{As}_{4}$ gas reservoir.

patterns in which atoms are rearranged once a particular configuration has become energetically unstable. It is necessary therefore to examine the available experimental information about such structural transitions, in conjunction with the total-energy versus chemical-potential curves, before attempting to propose particular models for the reconstructions.

The reconstruction believed to be stable on this surface is the Ga-vacancy geometry. There is strong evidence in its favor from LEED and $x$-ray diffraction experiments ${ }^{4,5}$ as well theoretical calculations. ${ }^{7}$ Since it is missing a $\mathrm{Ga}$ atom with respect to the ideal surface, this is an As-rich reconstruction. In the $\delta \mu=1.0 \mathrm{eV}$ region of Fig. 10 (Asrich environment) the vacancy is not actually the lowestenergy configuration. A review of the experiments dealing with this reconstruction reveals that in all the cases, the samples examined were actually annealed under high vacuum conditions. Taking into account the highly volatile nature of As (extremely low-sticking coefficient), we can conclude that the annealing process in high vacuum simulates a Ga-rich environment for the surface. At the Ga-rich region $(\delta \mu=-1.0 \mathrm{eV}$, Fig. 10) the Ga vacancy is indeed the lowest-energy configuration. This explains the apparent paradox of an As-rich geometry being the stable reconstruction of the Ga-terminated surface despite the high- (almost unity) sticking coefficient of $\mathrm{Ga}$ (see also Fig. 5, which shows the energy cost for removal of a $\mathrm{Ga}$ atom).

What is even more interesting is the indication that a phase transition to a different $(2 \times 2)$ reconstruction does take place as the As content of the surface is increased. Cho and Arthur ${ }^{25}$ observed that during MBE growth, an increase of the $\mathrm{As}_{2}$ arrival rate causes a change in the intensity of the half-order spots of the $(2 \times 2)$ diffraction pattern. They interpret this change as a transition between two different $(2 \times 2)$ reconstructions, since the half-order spots contain information about the atomic configuration inside the unit cell. Assuming that the Garich environment reconstruction is the Ga vacancy, the 
As-rich environment reconstruction could be the Astriangle geometry, predicted by our calculations. The fact that this geometry is not stable except in the As-saturated environment is consistent with our results indicating that the As triangle becomes the lowest-energy reconstruction only for $\delta \mu>0.2 \mathrm{eV}$.

Up to this point we have discussed the energetics of the surface reconstructions taking as reservoirs of $\mathrm{Ga}$ and $\mathrm{As}$ atoms, $\mathrm{Ga}$ bulk, and $\mathrm{As}_{2}$, respectively, in equilibrium with GaAs bulk. We will now examine the consequences of considering different atomic reservoirs. As a first step we consider $\mathrm{As}_{4}$ as the source of extra As atoms; this is a realistic alternative, as mentioned earlier, because of the stability of the $\mathrm{As}_{4}$ molecule and its presence in the MBE growth. Figure 10 exhibits the effect of using this alternative As reservoir: the dashed line gives the new upper bound of $\delta \mu$, and the intersection of this line with totalenergy curves gives the new energies at the As-rich environment. There is no qualitative change in the relative energies, although the As triangle is now lower than the Ga vacancy by only $0.3 \mathrm{eV}$.

We consider next the whole possible range of $\delta \mu$ without reference to any atomic reservoirs, except GaAs bulk. This range is obviously equal to the cohesive energy of bulk GaAs $(6.8 \mathrm{eV})$ : the As-rich environment consists of free As atoms in equilibrium with bulk GaAs and any additional $\mathrm{Ga}$ atoms needed for surface reconstructions have to be obtained by breaking GaAs bulk pairs (the converse holds for the Ga-rich environment). This situation is depicted graphically in Fig. 11. The zero of the relative chemical potential is chosen to be the same as in Fig. 10. The inset corresponds to the range of $\delta \mu$ and of the energy shown in Fig. 10. Only the lines which are important outside the latter range are shown; the rest of the totalenergy curves lie higher in energy and have slopes such that they will never become a lowest-energy configuration. The As-rich environment does not exhibit any features that are not already evident from Fig. 10. Namely, the As-triangle geometry dominates this regime and becomes more stable as the value of the relative chemical potential increases.

The Ga-rich environment shows a new phase transition at $\delta \mu=-1.4 \mathrm{eV}$ : at this value the buckled geometry, the $\mathrm{Ga}$ vacancy, and the $\mathrm{Ga}$ vacancy plus $\mathrm{Ga}$ substitutional become equal in energy. For still lower values of $\delta \mu$ the Ga vacancy plus Ga-substitutional geometry becomes the lowest-energy reconstruction and dominates the rest of the Ga-rich regime. In physical terms this indicates that as the $\mathrm{Ga}$ atom reservoir attains lower binding energy the most favorable reconstruction remains a vacancy geometry with one As atom replaced by a Ga atom. This is particularly interesting since the new geometry is actu-

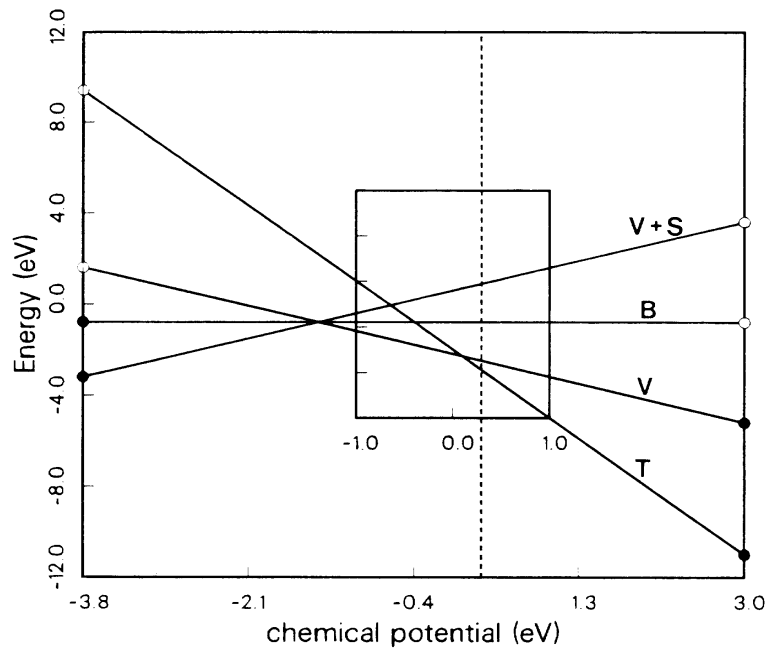

FIG. 11. Energy versus relative chemical potential $\delta \mu$ for the whole possible range of $\delta \mu$. Only the lowest-energy geometries are shown: $V+S$ is $\mathrm{Ga}$ vacancy plus Ga-substitutional geometry; $B$ is buckled geometry; $V$ is Ga-vacancy geometry; $T$ is As triangle geometry. The inset corresponds to the range of $\delta \mu$ and of the energy as shown in Fig. 10.

ally a Ga-rich surface. There is also some indirect evidence in support of this model: the synchrotron radiation experiments indicate that the atomic number of the atom in the proposed substitutional site is halfway between the atomic numbers of the type-III and type-V atoms. ${ }^{26} \mathrm{Fi}$ nally, we consider the character of this reconstruction: its stoichiometry $\left(S=-\frac{3}{4}\right)$ satisfies the condition for semiconducting character, but the substitution of an As atom by a $\mathrm{Ga}$ atom results in a metallic configuration (see Fig. 4). However, and $n$-doped sample would compensate for the electronic charge deficiency and produce a semiconducting surface with a gap of $\sim 0.62 \mathrm{eV}$. This leads to the conclusion that under highly Ga-rich conditions, and in particular, on an $n$-type doped substrate the most stable reconstruction is the Ga-vacancy plus Ga-substitutional geometry, described in Sec. III.

\section{ACKNOWLEDGMENTS}

This work was supported in part by U.S. Joint Services Electronic Program (JSEP) Contract No. DAAL-03-86K-0002. One of us (E.K.) would like to thank the IBM Thomas J. Watson Research Center (Yorktown Heights, NY) for its hospitality while portions of this work were carried out, and acknowledge partial support by IBM.
${ }^{1}$ A. Y. Cho and I. Hayashi, Solid-State Electron. 14, 125 (1971).

${ }^{2}$ R. Ludeke and L. Esaki, Phys. Rev. Lett. 33, 653 (1974).

${ }^{3}$ W. Ranke and K. Jacobi, Surf. Sci. 63, 33 (1977).

${ }^{4}$ S. Y. Tong, G. Xu, and W. N. Mei, Phys. Rev. Lett. 52, 1693 (1984).

5J. Bohr, R. Feidenhans'l, M. Neielsen, M. Toney, R. L.
Johnson, and I. K. Robinson, Phys. Rev. Lett. 54, 1275 (1985).

6J. R. Arthur, Surf. Sci. 43, 449 (1974).

7J. D. Chadi, Phys. Rev. Lett. 52, 1911 (1984).

${ }^{8}$ Recently proposed models [J. D. Chadi, Phys. Rev. Lett. 57 , 102 (1986); E. Kaxiras, Y. Bar-Yam, J. D. Joannopoulos, and 
K. C. Pandey, ibid. 57, 106 (1986)] have not yet been confirmed or disproved by other studies.

${ }^{9}$ Masahiko Nishida, J. Phys. C 14, 535 (1981).

${ }^{10}$ D. R. Hamann, M. Schlüter, and C. Chiang, Phys. Rev. Lett. 43, 1494 (1979).

${ }^{11}$ P. Hohenberg and W. Kohn, Phys. Rev. 136, B864 (1964); W. Kohn and C. J. Sham, ibid. 140, A1133 (1965).

${ }^{12}$ E. P. Wigner, Phys. Rev. 46, 1002 (1934).

13J. Ihm, A. Zunger, and M. L. Cohen, J. Phys. C 12, 4409 (1979).

${ }^{14}$ P. O. Löwdin, J. Chem. Phys. 19, 1396 (1951).

${ }^{15}$ J. Ihm and J. D. Joannopoulos, Phys. Rev. B 26, 4429 (1982).

${ }^{16}$ Hendrik J. Monkhorst and James D. Pack, Phys. Rev. B 13, 5188 (1976).

${ }^{17} \mathrm{R}$. Hultgren et al., Selected Values of the Thermodynamic Properties of the Elements (American Society of Metals, Metals Park, Ohio, 1973).

${ }^{18}$ A. R. Lubinsky, C. B. Duke, B. W. Lee, and P. Mark, Phys.
Rev. Lett. 36, 1058 (1976); P. J. Meyer, C. B. Duke, A. Paton, A. Kahn, E. So, J. L. Yeh, and P. Mark, Phys. Rev. B 19, 5194 (1979).

19J. D. Chadi, Phys. Rev. Lett. 41, 1062 (1978). James R. Chelikowsky and Marvin L. Cohen, Plys. Rev. B 20, 4150 (1979).

${ }^{20}$ R. W. Godby, M. Schluter, and L. J. Sham, Phys. Rev. Lett. 56, 2415 (1986); M. Hybertsen and S. G. Louie, ibid. 55, 1418 (1985).

${ }^{21}$ D. Haneman, Phys. Rev. 121, 1093 (1961).

${ }^{22}$ A. U. MacRae and G. W. Gobeli, J. Appl. Phys. 35, 1629 (1964).

${ }^{23}$ W. A. Harrison, J. Vac. Sci. Technol. 16, 1492 (1979).

${ }^{24}$ E. Kaxiras, K. C. Pandey, Y. Par-Yam, and J. D. Joannopoulos, Phys. Rev. Lett. 56, 2819 (1986).

${ }^{25}$ A. Y. Cho and J. R. Arthur, Prog. Solid State Chem. 10, 157 (1975).

${ }^{26}$ I. K. Robinson (private communication). 